\title{
COMPETENCIA LINGÜIISTICA Y CONCIENCIA INTERCULTURAL A TRAVÉS DEL INTERCAMBIO VIRTUAL
}

\author{
Martha García Chamorro, ${ }^{39}$ Lourdes Rey Paba ${ }^{40}$ y Nayibe Rosado Mendinueta. ${ }^{41}$
}

\section{RESUMEN}

El presente artículo trata de cómo la participación en actividades de intercambio virtual con herramientas tecnológicas, apoya el desarrollo de competencias lingüísticas y de la conciencia intercultural de los estudiantes de lengua extranjera del Instituto de Idiomas de la Universidad del Norte y del Colegio Richmond Senior High en Rockingham, Richmond, Carolina del Norte. Además, describe la experiencia, las etapas de la misma y algunos resultados obtenidos a partir de la aplicación de una encuesta a los estudiantes participantes. En términos generales, parece haber una relación positiva entre la participación de los estudiantes en esta experiencia de intercambio y el desarrollo de sus competencias lingüísticas y de su conciencia intercultural.

Palabras clave: competencias lingüísticas, conciencia intercultural, intercambio virtual.

\begin{abstract}
This article reports the experience of students from the Language Institute at Universidad del Norte and students from Richmond Senior High en Rockingham, Richmond, North Carolina who participated in virtual exchange project and the relationship of this participation with the development of their linguistic competence and their cultural awareness. The article describes the experience, its stages and some of the results obtained form a survey applied to participating students. In general terms, the results seem to suggest that there is a positive relationship between the participation in this type of virtual exchange activities and their development of linguistic competence and their intercultural awareness.
\end{abstract}

Key words: linguistic competence, intercultural awareness, virtual exchange.

Recibido: 1 de octubre de 2009

Aceptado: 25 de mayo de 2010

39 Licenciada en Lenguas, Universidad del Atlántico. Especialista en la Enseñanza del Inglés, Universidad del Norte. Candidata a Magíster en Educación con énfasis en inglés, Universidad del Norte. Diplomado en Nuevas Tecnologías aplicadas a la Educación. Docente catedrática del programa de Pregrado y Extensión del Instituto de Idiomas de la Universidad del Norte. magarcia@uninorte.edu.co, Barranquilla, Colombia.

40 Máster en Educación con Énfasis en la Enseñanza del Inglés, Especialización en la misma área, ambos títulos de la Universidad del Norte donde labora actualmente en el cargo de Coordinadora Académica del Instituto de Idiomas. Es tutora certificada de ESOL Cambridge para el programa de Desarrollo Profesoral ICELT. arey@uninorte.edu.co.

41 Licenciada en Lenguas, Universidad del Atlántico. Especialista en la Enseñanza del Inglés, Universidad del Norte. Magistra en Educación, Universidad del Norte. Docente tiempo completo del Instituto de Idiomas de la Universidad del Norte. Barranquilla, Colombia.nrosado@uninorte.edu.co 


\section{INTRODUCCIÓN}

Lapresente experiencia atiende a la necesidad que tienen los estudiantes de aumentar su competencia comunicativa en lengua extranjera y apoya este aprendizaje con el uso de la tecnología.

Según Richards (1995) la implementación de actividades de intercambio virtual para estimular un contacto más cercano con culturas extranjeras ha motivado a estudiantes en otros contextos a aprender idiomas extranjeros y a conocer culturas diferentes a las propias. En esta línea, el Instituto de Idiomas de la Universidad del Norte, a través del uso de las tecnologías de la comunicación, busca fortalecer el desarrollo lingüístico e intercultural de los estudiantes con la intención de formar ciudadanos críticos capaces de participar en el proceso permanente de construcción de la democracia y de la armonía entre naciones ya que al expandir su competencia comunicativa en lengua extranjera tienen mayor probabilidad de desplegar actitudes de aceptación de las culturas y subculturas asociadas a dicha lengua.

De acuerdo con Lee (1999) las actividades de intercambio virtual hacen del acto de comunicarse en inglés (o en otra lengua extranjera) no sólo un problema teórico, sino uno práctico. El requerimiento de resolver ese problema práctico de comunicación puede ser un gran motivo para que los estudiantes se esfuercen en construir mensajes gramaticalmente correctos que comuniquen sus pensamientos. Los intercambios virtuales se constituyen en un gran incentivo para el uso auténtico de la lengua en tareas reales de comunicación.

En esta experiencia entre dos grupos de estudiantes de dos contextos culturales y lingüísticos diferentes pretendimos establecer relaciones entre la utilización de actividades de intercambio virtual y el desarrollo de su nivel de competencia lingüística así como mayores niveles de conciencia de la cultura propia y la extranjera. Para tal efecto, utilizamos una encuesta en la que se recoge información de los participantes y sus propias interacciones en la plataforma tecnológica. Luego, se procedió al análisis de esta información desde una mirada cualitativa que nos permitiera interpretar y relacionar los datos. Se encontró que hay relaciones positivas entre la experiencia de intercambio virtual y un mayor nivel de competencia lingüística y de conciencia intercultural en los estudiantes participantes en los dos contextos.

A continuación se presentará la metodología con la cual se desarrolló la experiencia, algunos resultados y conclusiones que se pueden derivar de la información analizada, y finalmente algunas recomendaciones a tener en cuenta para futuras implementaciones de experiencias similares.

\section{METODOLOGÍA}

Para llevar a cabo y sistematizar la experiencia, ésta se organizó en varios momentos y etapas y se recogió información de los participantes que nos permitiera valorar aciertos y desaciertos que se convirtieran en aprendizajes a considerar para futuras implementaciones.

Siendo esta una experiencia de intercambio virtual, un primer momento fue la determinación de los aprendices y docentes que participarían de la experiencia. En este caso los dos 
grupos fueron importantes; los docentes que dieron soporte a la organización, planeación e implementación de las acciones que llevaron esta experiencia a término y los aprendices que mostraron una actitud positiva.

Los participantes fueron dos grupos de estudiantes y sus respectivos docentes. Un grupo de estudiantes de la Universidad del Norte en Barranquilla, Colombia y otro grupo de estudiantes del Colegio Richmond Senior High en Rockingham, Richmond, Carolina del Norte. Los estudiantes de la Universidad provenían de primeros semestres de diferentes programas académicos con un nivel de inglés de usuario básico o nivel A1 según la clasificación del Marco de Referencia Europeo para la enseñanza y el aprendizaje de lenguas extranjeras. Son estudiantes con edades entre 17 y 19 años. Los estudiantes del Colegio Richmond Senior High en Rockingham, Richmond, Carolina del Norte eran estudiantes de grados 10, 11 y 12 con un nivel de español de usuario básico o nivel A1 según la clasificación del Marco de Referencia, con edades entre 16 y 18 años. Las dos docentes son colombianas; una profesora de inglés como lengua extranjera y la otra de español como lengua extranjera. Ambas han mostrado un constante interés por la utilización de las nuevas tecnologías de la comunicación como apoyo al aprendizaje de las lenguas.

Etapa 1 de planeación tecnológica: las profesoras participantes planearon una serie de tareas que involucraran la interacción entre sus estudiantes en el idioma extranjero por lo cual era necesario determinar las herramientas tecnológicas apropiadas. Para la toma de decisiones tecnológicas se contó con el apoyo de la Unidad de Nuevas Tecnologías de la Universidad del Norte y al Centro de Informática. En esta etapa se definió utilizar la plataforma del Catálogo Web que la Universidad del Norte usa para complementar las clases presenciales. El Webct cuenta con herramientas de comunicación como correo electrónico, foro y chat. El uso de estas herramientas apoyaría las "conversaciones" (nuestras comillas) que se desarrollarían a miles de kilómetros de distancia entre personas que nunca se habían conocido excepto en la Internet. Estas conversaciones tenían como tema de fondo actividades sociales, y las actividades sociales tienden a estimular a la interacción entre los estudiantes (Nagel, 1999).

Como los estudiantes deseaban "conocer" (nuestras comillas) a sus pares se decidió complementar la plataforma Webct con el uso del skype. El skype se seleccionó para que los estudiantes pudieran interactuar oralmente (habla y escucha) con estudiantes nativos de la lengua de estudio y así pudieran probar el grado de desarrollo de sus habilidades y estimular su proceso de aprendizaje. Además, el hecho de permitirle a los estudiantes ver a la persona con la cual se están comunicando les facilitaría desarrollar su propio lenguaje en el idioma nuevo, aparte de que es un factor altamente motivante. Se autorizó entonces la instalación de skype en los computadores del Laboratorio del Instituto de Idiomas.

El último paso para culminar esta etapa fue crear un grupo en Webct en donde se incluyeron tanto los estudiantes del Instituto de Idiomas como los del Colegio Richmond para dar inicio a la actividad académica propiamente dicha. 
Etapa 2 de diseño: una vez se establecieron las herramientas tecnológicas para el uso en las actividades de comunicación oral y escrita, Catalogo Web y Skype, y teniendo en cuenta que las lenguas objetivo (español e inglés) se enseñan con un enfoque comunicativo, es decir que la lengua es el medio para expresar ideas y puntos de vista y no un listado de funciones gramaticales, las profesoras iniciaron el diseño de las actividades a realizar y los tiempos en los cuales se debían completar así como la selección de los estudiantes que participarían en la experiencia.

Las profesoras escogieron una serie de temas correspondientes a sus currículos y que se desarrollaran tanto en el programa de inglés como en el de español. También definieron la metodología para la actividad y el número de encuentros entre los estudiantes. Establecieron un cronograma de realización de las tareas y las herramientas que serían utilizadas para cada caso haciendo un balance entre actividades sincrónicas y asincrónicas. Seleccionaron a los estudiantes que por su horario se les facilitara el contacto con sus pares (había que considerar la diferencia horaria y la posibilidad de realizar algunas actividades sincrónicas durante las horas en las cuales tenían clases de lengua) y establecieron grupos o pares de trabajo. Una vez organizada esta parte logística se dio inicio al intercambio virtual.

Etapa 3 de desarrollo: se estableció un número de cuatro encuentros con un tema como excusa para la comunicación entre los participantes. Dicho tema apareció dentro de la herramienta de Medios de Comunicación "Foro" en Webct. El primer encuentro se estableció con el nombre de: Mi Información Personal 1, como punto de inicio de las relaciones entre los estudiantes de Colombia y los de los Estados Unidos, y en el cual se dieron a conocer de manera sencilla.

Un segundo encuentro se dio a través del chat, otra de las herramientas de Medios de Comunicación de webct, en el cual ahondaron acerca de su información personal. En ellos los estudiantes de ambos grupos tomaban turnos de aproximadamente diez minutos para interactuar en cada idioma. Esta experiencia les permitió adoptar el papel de par en donde se corregían errores de gramática, de construcción de oraciones y de vocabulario. Los diálogos en chat fueron grabados para su posterior estudio.

Un tercer encuentro en el Foro (webct) con el tema Mi Información Personal 2, en el cual los estudiantes realizaron una presentación en power point que incluía foto y que abarcaba aspectos más detallados de su vida personal y sus intereses. Una vez publicadas las presentaciones, los estudiantes realizaron preguntas en el idioma de estudio para sus compañeros.

Finalmente, un quinto encuentro se realizó utilizando Skype para intercambiar preguntas de información personal y poner a prueba sus habilidades orales en habla y escucha. Esta herramienta favoreció el aprendizaje de la lengua en un contexto real y les permitió a los estudiantes usar diferentes estrategias de repetición, de aclaración de información, propias de su nivel de lengua, para lograr enviar su mensaje. 
Etapa 4 de recolección de información: al finalizar la experiencia se aplicó una encuesta a los 120 estudiantes que participaron de esta experiencia en los dos contextos. La encuesta diseñada por las docentes que lideraron el intercambio pretendía explorar aspectos como: el interés generado por las actividades y el tiempo dedicado a ellas, el aprendizaje sobre la cultura de los pares y la propia, utilización de estrategias para el abordaje de los temas, y la percepción de aprendizaje de la lengua específicamente la competencia lexical o de vocabulario (ver anexo 1). Se hicieron dos versiones de la encuesta: una en inglés y otra en español. La encuesta se envió por correo electrónico en su idioma materno.

A continuación los resultados de la información obtenida durante la realización de la experiencia, específicamente a través de la encuesta aplicada a la población de estudiantes participantes de este intercambio virtual.

\section{RESULTADOS}

Nuestro objetivo con la sistematización y el análisis de esta experiencia era la de establecer relaciones entre la utilización de actividades de intercambio virtual y el desarrollo de su nivel de competencia lingüística así como mayores niveles de conciencia de la cultura propia y la extranjera. A continuación discutiremos la información obtenida. El número total de estudiantes participantes fue de 120 de los cuales 50 completaron la encuesta. 29 del grupo de Rockingham High School y 21 del grupo de la Universidad del Norte.

En la pregunta 1 cuando se indagó si actividades como las de este proyecto incentivan el aprendizaje del idioma extranjero, el $80 \%$ de los estudiantes de Rockingham High School y el 100\% de los estudiantes de la Universidad del Norte estuvo de acuerdo.

En la pregunta 2, con respecto a si aprendieron algo de la cultura de sus compañeros del otro contexto cultural, el $90 \%$ de los estudiantes Rockingham High School y el 100\% de los estudiantes de la Universidad del Norte estuvo de acuerdo. En la pregunta 3 también relacionada con el contexto exploraba si las actividades les ayudaron a afirmar su propia identidad cultural. El 85\% de los estudiantes de Rockingham High School y el $82 \%$ de los estudiantes de la Universidad del Norte estuvieron de acuerdo.

La pregunta 4 en la que indagaba por las similitudes con sus pares en la manera en que habían abordado los temas, el 71\% de los estudiantes de Rockingham High School y el $81 \%$ de los estudiantes de la Universidad del Norte estuvieron de acuerdo con que había similitudes en el abordaje de los temas. Al preguntárseles por las diferencias en la pregunta 5 (en la que se les preguntó si encontraron diferencias con sus pares en la manera en que habían abordado los temas), El 68\% de los estudiantes de Rockingham High School y el 60\% de los estudiantes de la Universidad del Norte estuvieron de acuerdo en que había diferencias.

En la pregunta 6, se abordó el tema del tiempo dedicado a leer los mensajes de los compañeros del otro curso. El 88\% de los estudiantes de Rockingham High School y el 98\% de los estudiantes de la Universidad del Norte estuvo de acuerdo con que habían dedicado tiempo. 
Cuando se les indagó si habían aprendido algo nuevo -vocabulario, nuevas frases- con su participación en el proyecto, El 92\% de los estudiantes de Rockingham High School y el 85\% de los estudiantes de la Universidad del Norte estuvo de acuerdo en que habían aprendido vocabulario y frases nuevas.

A continuación se presentan las tablas con el consolidado de los resultados de cada uno de los contextos. Los números en la parte inferior horizontal indican la cantidad de la pregunta y los números del lado izquierdo y vertical indican los porcentajes en cada una de las preguntas.

Gráfica 1. Resultados de encuestas Estudiantes Rockingham High School

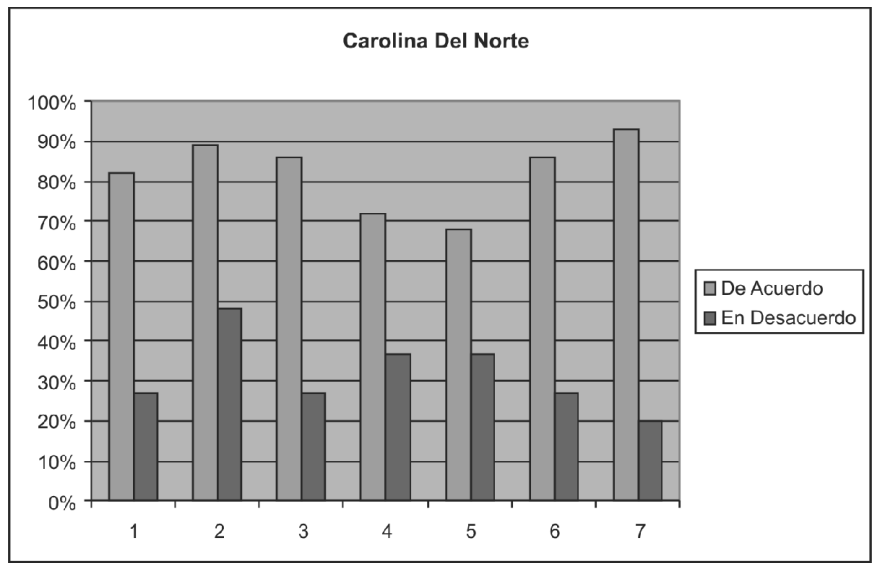

Gráfica 2. Resultados de encuestas Estudiantes Universidad del Norte

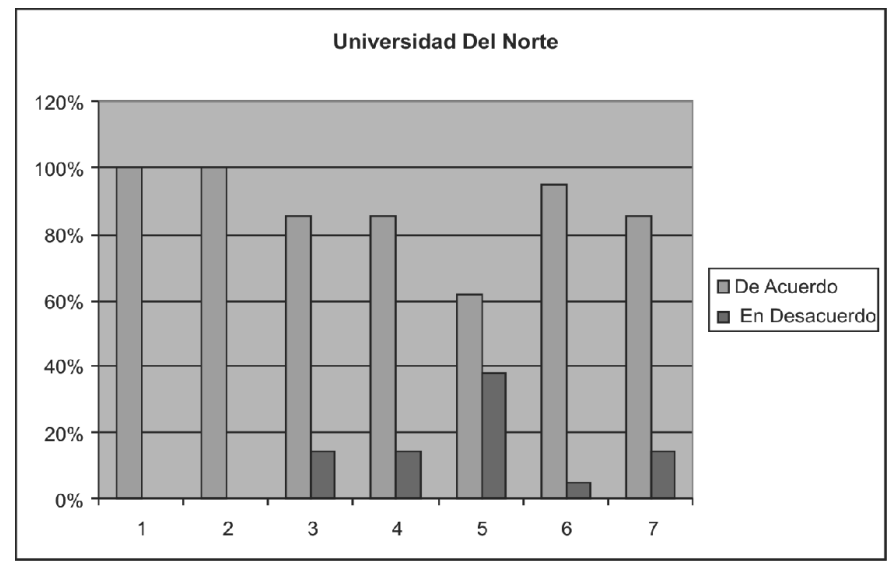




\section{Discusión de Resultados}

Según los resultados de la breve encuesta aplicada se puede interpretar lo siguiente:

Actividades incentivan el aprendizaje: las respuestas de los estudiantes parecen sugerir que la utilización de actividades de intercambio contribuyen a incentivar su aprendizaje del idioma extranjero. Esto confirma la información de autores que han investigado estos temas de manera más extensa y en otros contextos. ( Lee, 1999; Nagel,1999, Richards, 1995; Castells, 2000; Dreyfus, 2003; Duart y Sangrá ,2000; García, 2003). Se reafirma, además, la dedicación a las actividades de intercambio, que es muy alta en ambos casos.

Aprendizaje sobre la cultura de compañeros: este aprendizaje sobre la cultura del otro, el descubrimiento de la otredad como concepto esencial para construir la sociedad en la que nos movemos parece haber sido reconocida a través de las actividades de intercambio propuestas en esta experiencia. Se puede contrastar este punto con el que tiene que ver con la afirmación de identidad cultural: de manera paralela al descubrimiento del otro, de la idea del otro, está la afirmación de la propia identidad. Es decir, debe conocerse para poder explicarle al otro que sabe poco sobre su cultura. Estos dos aspectos son reconocidos por los estudiantes según las respuestas obtenidas en las encuestas y en los mismos productos e interacciones que llevaron a cabo durante la experiencia.

Similitudes y diferencias el abordaje de los temas:dadas las diferencias de contextos de estudio, culturales y socioeconómicos, la información de la encuesta parece sugerir que hay diferencias en la manera como los dos grupos abordan los temas a tratar en las actividades. Pero de igual manera, hay similitudes dadas por las edades y el hecho de ser estudiantes de lenguas extranjeras que experimentan situaciones similares en este proceso. Al analizar las interacciones en el Webct se puede notar que los estudiantes tomaron en cuenta aquellos aspectos que compartían por su edad e intereses e igualmente aquellos que los hacían diferentes, pues se desenvuelven en medios y contextos regionales disímiles. Las discusiones llevadas a cabo por las profesoras después de las actividades, impulsaron su reflexión acerca de las variantes que influyen en el desarrollo cultural de los seres humanos, no obstante, fenómenos como la globalización permite puntos que faciliten el encuentro y socialización de diferentes culturas, de experiencias de vida, de la historia, que, entre otros aspectos, singularizan a los grupos de seres humanos.

Aprendizaje lingüístico: los resultados de la encuesta permitieron establecer el desarrollo del nivel lingüístico lexical. En el análisis de sus interacciones en WEBCT se notó, además, un manejo de sintaxis adecuada, de pronunciación y significados de vocablos adecuados y la entonación requerida para la formulación de preguntas. Todo lo anterior estimuló el interés de los estudiantes por participar de las actividades. Igualmente, a nivel afectivo, se observó un incremento en el nivel de confianza que adquirieron los estudiantes en cuanto a su aprendizaje del idioma, para ambos grupos la participación y culminación de las actividades desarrollaron en ellos un sentido de satisfacción, pues pudieron medir el alcance de lo aprendido y su relevancia en la comunicación. 
Esta satisfacción se puede evidenciar en sus comentarios en la encuesta. Por ejemplo, un estudiante dice "Esta fue una excelente experiencia de aprendizaje para mí. Disfruté el conocer nuevas personas de otro país. También comprendí las varias diferencias en mi país y otros. Por eso, le agradezco." (Estudiante de Richmond High School); otro dice "Me encantó aprender acerca de otro país. Fue muy interesante" (Richmond High School ); y otro añade "... ansiaba venir a mi clase de Español y ver lo que tenían que decir acerca de mí y mi clase." (Richmond High School).

Además de todo lo anterior, un aspecto importante que cabe destacar es el hecho de que esta experiencia de intercambio se llevó a cabo en un ambiente tecnológico seguro ya que las interacciones se dieron bajo unas características académicas que brindaron un espacio seguro de socialización, con personas diferentes, sin correr ninguno de los riesgos que pueden encontrar en las páginas comerciales.

\section{CONCLUSIONES Y RECOMENDACIONES}

Los resultados parecen sugerir que hay una relación positiva entre la participación en actividades de intercambio virtual y el desarrollo de las competencias lingüísticas de los estudiantes participantes. Su expansión del vocabulario, el uso apropiado del mismo, así como la utilización de una sintaxis adecuada a la tarea de comunicación que debían realizar, dan indicios de este avance. Con respecto al desarrollo de su conciencia intercultural, se puede concluir que la participación en esta experiencia ayudó a que cobraran conciencia tanto de la cultura de otro, del par con el que se comunicaban en la lengua extranjera, como de sí mismos como portadores de una cultura propia y única. Esta conciencia es determinante para la construcción permanente del proceso de la democracia y de la armonía entre naciones ya que en conjunción con su creciente competencia comunicativa en lengua extranjera, aumenta la probabilidad de desplegar actitudes de aceptación de las culturas y subculturas asociadas con esta lengua.

Teniendo en cuenta los resultados positivos de la evaluación y el interés que causó el proyecto de intercambio en ambos grupos, la institución se ha trazado algunos lineamientos para el mejoramiento, extensión y realización de las actividades que se resumen a continuación:

- Continuar con la estructuración de más actividades que permitan el intercambio de información cultural, como por ejemplo, la socialización de los elementos que componen la convivencia ciudadana en la región de cada grupo y el sistema social que caracteriza a la región.

- Aumentar el número de participaciones a través de Skype como herramientas para el desarrollo de los aspectos formales de la lengua en cuanto a las habilidades de habla y escucha que permitan el desarrollo igualmente de la interacción. 
- Buscar establecer contacto con estudiantes de niveles superiores de la lengua para realizar las discusiones de las competencias culturales y desarrollo del pensamiento crítico en la lengua de estudio: español e inglés.

- Aumentar los puntos de contacto en la parcelación de las actividades del programa utilizando la herramienta de Foro del catalogo Web.

Esta experiencia de intercambio virtual entre dos grupos de estudiantes de lengua extranjera incentivó su interés por el aprendizaje de la lengua y mostró una relación positiva entre la experiencia de intercambio virtual y un mayor nivel de competencia lingüística y de conciencia intercultural en los estudiantes participantes en los dos contextos. Por otro lado, los profesores participantes directos y otros profesores de ambas instituciones han incrementado su motivación por seguir participando en este tipo de proyectos. Tal interés ha desembocado en el incremento de los recursos tecnológicos del laboratorio informático de lenguas dentro de la Universidad del Norte y en el Colegio Richmond así como la mejoría de los recursos disponibles para el apoyo de este tipo de experiencias.

Hay muchas limitaciones en esta experiencia al considerar sólo las encuestas como fuente de información, y por lo tanto, surgen preguntas e inquietudes para continuar la exploración de este tema: siendo la interacción mayormente escrita, ¿cómo podemos incrementar el desarrollo armonioso de las otras habilidades lingüísticas y comunicativas?; ¿la motivación percibida en los estudiantes es dada por la novedad de la actividad o se podrá mantener si se proponen actividades secuenciales a través de sus proceso de aprendizaje de la lengua? Estas y otras preguntas que surgen en estas experiencias podrán dar pie para que docentes inquietos por el aprendizaje de las lenguas y el papel que juegan las tecnologías de la comunicación en este proceso podamos seguir investigando.

\section{REFERENCIAS BIBLIOGRÁFICAS}

CASTELLS, M. (2000). La Era de la Información Vol.1 La sociedad red. Alianza Editorial Madrid, España. Segunda Edición

DREYFUS, H. L.( 2003). Acerca del Internet. Colecciones Nuevas Tecnologías y Sociedad. Editorial UOC. Barcelona, España

DUART, J.M.. y SANGRÁ A. (compiladores). (2000). Aprender en la virtualidad. BarcelonaEspaña Edicionesde la Universitat Oberta de Catalunya. Gedisa Editorial.

GARCÍA, J. (2003). Entornos virtuales de aprendizaje. ¿Un sistema Didáctico? Revista digital de Educación y Nuevas tecnologías. Contexto Educativo. Número 28. http:// contexto-educativo.com.ar/2003/4/nota-06.htm.

HOWARD, P. N y JONES, S. (2005). Sociedad on line Internet en contexto. Colección Nuevas tecnologías y sociedad Editorial UOC Barcelona, España. 
JÁNICA, D. (2006). Analysis of the Characteristics of a Multimedia Material for an Effective Transcultural Learning for level 7 in the English Undergraduate Program at Instituto de Idiomas in the Universidad del Norte. Thesis Dissertation. Universidad del Norte. Barranquilla, Colombia.

LEE, K. (1999). Energizing the ESL/EFL Classroom through Internet activities. The Internet TESL Journal.

NAGEL, P.S. (1999). Email in the Virtual Esl/Efl Classroom. The Internet Tesl Journal http://Aithech.Ac.Jp/Iteslj/Articles/Nagel-Email.html

RICHARDS, J. (1995). "Easier said than done: an insider's account for a text project in Hidalgo". A. D. Hall and G. Jacobs (Eds). Getting Started: Materials Writing. Singapore: Seamo Regional Language Centre. 


\section{ANEXO 1.}

\section{ENCUESTA A ESTUDIANTES}

1. ¿Crees que actividades como las de este proyecto incentivan el aprendizaje del inglés/ español?

2. ¿Aprendiste algo de la cultura de tus compañeros de Rockingham High School/Universidad del Norte?

3. ¿Sientes que estas actividades te ayudan a afirmar tu identidad cultural colombiana/ americana?

4. ¿Encontraste similitudes en tu forma de abordar los temas y las de ellos?

5. ¿Encontraste diferencias entre tu forma de abordar los temas y la de ellos?

6. ¿Dedicaste tiempo a leer los mensajes de los compañeros del otro curso?

7. ¿Aprendiste algo nuevo -vocabulario, nuevas frases- con tu participación en el proyecto?

Comentarios: 